\title{
The targeted delivery of IL 17 to the mouse tumor neo-vasculature enhances angiogenesis but does not reduce tumor growth rate
}

Journal Article

Author(s):

Pasche, Nadine; Frey, Katharina; Neri, Dario (1)

Publication date:

2012-03

Permanent link:

https://doi.org/10.3929/ethz-b-000044543

Rights / license:

$\underline{\text { In Copyright - Non-Commercial Use Permitted }}$

Originally published in:

Angiogenesis 15(1), https://doi.org/10.1007/s10456-011-9239-8 


\title{
The targeted delivery of IL17 to the mouse tumor neo-vasculature enhances angiogenesis but does not reduce tumor growth rate
}

\author{
Nadine Pasche $\cdot$ Katharina Frey $\cdot$ Dario Neri
}

Received: 31 May 2011/Accepted: 20 October 2011/Published online: 4 November 2011

(C) Springer Science+Business Media B.V. 2011

\begin{abstract}
There has been a long controversy as to whether interleukin-17 (IL17) has an impact on tumor growth. In order to assess whether IL17 may affect tumor growth, it would be convenient to achieve high levels of this pro-inflammatory cytokine at the tumor neo-vasculature, since IL17 is known to promote angiogenesis. Here, we have generated and tested in vivo a fusion protein, consisting of the F8 antibody (specific to the alternatively spliced EDA domain of fibronectin, a marker of angiogenesis) and of murine IL17 (mIL17). The resulting immunocytokine (termed F8-mIL17) was shown to selectively localize at the tumor neo-vasculature and to vigorously promote tumor angiogenesis, without however reducing or enhancing tumor growth rate both in immunocompetent and in immunodeficient mice.
\end{abstract}

Keywords Immunocytokines · IL17 ·

Vascular tumor targeting · EDA domain of fibronectin

\section{Introduction}

Interleukin 17 (IL17) is a homodimeric cytokine mostly expressed by Th17 cells [1]. It acts mainly as proinflam-

Electronic supplementary material The online version of this article (doi:10.1007/s10456-011-9239-8) contains supplementary material, which is available to authorized users.

N. Pasche $\cdot$ K. Frey $\cdot$ D. Neri $(\bowtie)$

Department of Chemistry and Applied Biosciences, Institute of Pharmaceutical Sciences, ETH Zurich, Wolfgang-Pauli-Strasse 10, 8093 Zurich, Switzerland e-mail: neri@pharma.ethz.ch matory cytokine and was found to play a major role in inflammation and autoimmune diseases [2-5]. In addition, IL17 stimulates angiogenesis [6].

It is controversial whether IL17 favors or inhibits tumor growth [1]. Tumor cells transfected with the IL17 gene grew faster than untransfected cells when implanted in immunodeficient mice [6,7], but similar experiments performed with IL17 in immunocompetent mice gave ambiguous results [8, 9]. Moreover, MC38 tumor implantation in IL17 knockout mice led to faster tumor growth in one laboratory [10] but not in a second laboratory, where the experiments were repeated [11].

Our group has demonstrated the possibility to deliver cytokines to the tumor environment using fusion proteins based on human monoclonal antibodies specific to splice isoforms of fibronectin [12-18] and of tenascin-C [19, 20]. In particular, the F8 antibody, specific to the alternatively spliced domain of fibronectin EDA (a marker of angiogenesis [21]) was shown to selectively localize at the tumor neo-vasculature following intravenous administration [22, 23].

As knock-out mouse models and tumor cells overexpressing IL17 offer little control over the specific location of IL17 expression within the tumor mass, we reasoned that fusion of IL17 to an antibody capable of selective localization on the tumor neo-vasculature could allow us to more precisely investigate the function of this cytokine when deposited at high local concentration on tumor blood vessels.

Here, a fusion protein consisting of the F8 antibody and of murine IL17 (F8-mIL17) was shown to selectively localize at the tumor neo-vasculature and to vigorously promote tumor angiogenesis, without however impacting on tumor growth rate. 


\section{Materials and methods}

Cell lines

CHO-S cells and the F9 murine teratocarcinoma cell line (CRL-1720, ATCC) were cultivated as described before [24]. NIH 3T3 fibroblasts were cultured in RPMI 1640 (Gibco) containing 10\% FCS (Invitrogen), $2 \mathrm{mM}$ ultraglutamine (Lonza), $1 \mathrm{mM}$ sodium pyruvate (Gibco) and $50 \mu \mathrm{M} \beta$-mercaptoethanol (Gibco) and incubated at $37^{\circ} \mathrm{C}$ and $5 \% \mathrm{CO}_{2}$.

Cloning, expression, purification and characterization of F8-mIL17

For the cloning of F8-mIL17, containing a NheI restriction site upstream of the leader sequence, the $(\mathrm{scFv}) \mathrm{F} 8$ gene was PCR amplified from the previously described clone F8-SIP [23] using primers RS9 (5'-ctagctagcgtcgaccatgg gctggagcctgat- $\left.3^{\prime}\right)$ and NP28 $\left(5^{\prime}\right.$-acctccaccgccagaaccacttccg cctgatttgatttccaccttggtccettg- $\left.3^{\prime}\right)$ appending a 10 amino acid linker at the C-terminus of the antibody moiety. The mIL17 gene (Source BioScience) was amplified by PCR with primers NP30 (5'-tcaggcggaagtggttctggcggtggaggtgcagcgat catccctcaaagctc- $\left.3^{\prime}\right)$ and NP24 (5'-tcgataagcttcattaggetgcc tggcggacaatcgag- $3^{\prime}$ ) introducing two stop codons and a HindIII restriction site. The $\operatorname{scFv}(\mathrm{F} 8)$ and the mIL17 genes were PCR-assembled using primers RS9 and NP24. The double-digested NheI/HindIII assembly product was cloned into pcDNA3.1(+) (Invitrogen) vector. A stable CHO-S cell line growing in suspension and expressing F8-mIL17 was generated as previously reported [24]. F8-mIL17 was purified from the cell culture medium by protein A affinity chromatography. F8-mIL17 was analyzed by SDS PAGE, size exclusion chromatography (Superdex200 10/300 GL, GE Healthcare) and BIAcore on an EDA coated sensor chip.

\section{Bioactivity assay}

The biological activity of F8-mIL17 was determined by its ability to induce IL6 production in NIH 3 T3 fibroblasts. $4 \times 10^{4}$ cells were seeded in 96-well plates in medium containing serial dilutions of F8-mIL17 or recombinant mIL17 (standard, Chemie Brunschwig) or culture medium (negative control). After $48 \mathrm{~h}$, cytokine expression in supernatants was determined with Mouse IL6 ELISA Ready-SET-Go! (BD Biosciences).

Quantitative biodistribution studies

The in vivo targeting performance of F8-mIL17 was evaluated by biodistribution analysis as described before [22]. F8-mIL17 was radioiodinated and injected into the tail vein of female 129/SvPas (Taconic) (14.5 $\mu \mathrm{g}$ radiolabeled protein or $7.5 \mu \mathrm{g}$ radiolabeled plus $60 \mu \mathrm{g}$ unlabeled protein) or Balb/c nude mice (Charles Rivers) $(14.5 \mu \mathrm{g}$ radiolabeled protein) bearing s.c. implanted F9 tumors [22-25]. Mice were sacrificed $24 \mathrm{~h}$ after injection.

Syngeneic tumor mouse models

Tumor bearing mice were obtained by subcutaneous injection of F9 cells $\left(10^{7}\right)$ in 12 week-old female 129/SvPas or Balb/c nude mice [22, 24]. Four days after tumor implantation, mice were grouped and injected into the lateral tail vein three times every $48 \mathrm{~h}$ with F8-mIL17 $(100 \mu \mathrm{g})$ or saline. Mice were monitored daily and tumor growth was measured with a caliper using the formula: volume $=$ length $\times$ width $^{2} \times 0.5$. Animals were sacrificed when tumor volumes reached $2000 \mathrm{~mm}^{3}$. The experiment was repeated in immunocompetent mice, which were sacrificed 3 days after last injection. Tumors were excised, embedded in cryoembedding medium (ThermoScientific) and stored at $-80^{\circ} \mathrm{C}$. Experiments were performed under a project license granted by the Veterinäramt des Kantons Zürich, Switzerland (169/2008).

Immunofluorescence analysis on tumor sections

Immunofluorescence staining of cryostat sections $(10 \mu \mathrm{m})$ of F9 tumors was performed essentially as described [22] using as primary antibodies rat anti F4/80 (macrophages, Abcam), rat anti CD45 (leukocytes, BD Biosciences), rat anti CD31 (endothelial cells, BD Biosciences), rat anti CD4 (CD4 T cells), rat anti CD8 (CD8 T cells), rabbit anti Asialo/GM1 (NK cells, Wako Pure Chemical Industries) antibodies and biotinylated F8-mIL17. Anti rat IgG-AlexaFluor488, anti rat IgG-AlexaFluor594, anti rabbit IgG-AlexaFluor488, as well as streptavidin-AlexaFluor488, were used as secondary reagents for microscopic detection. Images were obtained with an Axioskop2 mot plus microscope (Zeiss), the staining areas were analyzed using ImageJ software and expressed as percentage of measurement area.

\section{Results and discussion}

We cloned and expressed in mammalian cells a fusion protein consisting of $\mathrm{scFv}(\mathrm{F} 8)$ sequentially fused to a murine IL17 monomer (Fig. 1a). The two mIL17 monomers drive the disulfide-linked covalent homodimerization of the fusion protein, forming a stable immunocytokine termed "F8-mIL17" (Fig. 1b). F8-mIL17 was purified by protein A chromatography, exhibited a homogeneous profile in size-exclusion chromatography with a retention time 
corresponding to the expected dimeric structure (Fig. 1c), ran as a covalent homodimer in SDS-PAGE in nonreducing conditions and as a monomer in reducing conditions (Fig. 1d). F8-mIL17 was shown to retain the binding affinity of the parental antibody in a BIAcore assay (Fig. 1e) [23] and to be fully active in an in vitro cytokine production assay (Fig. 1f). A radioiodinated preparation of F8-mIL17 was analyzed in a quantitative biodistribution study in immunocompetent mice bearing subcutaneous F9 tumors, a model frequently used to study vascular-targeting antibody-based fusion proteins [22-25], confirming a preferential accumulation in the tumor mass [4.3\% injected dose per gram $(\% \mathrm{ID} / \mathrm{g})$ at $24 \mathrm{~h}]$, compared to normal organs [tumor to blood ratio of 14:1 (Fig. 1g)]. Similar results were obtained in athymic mice (Fig. 1g). As expected and in line with previous experiments based on the F8 antibody [23], the F8-mIL17 immunocytokine localized around tumor blood vessels (Fig. 1h).

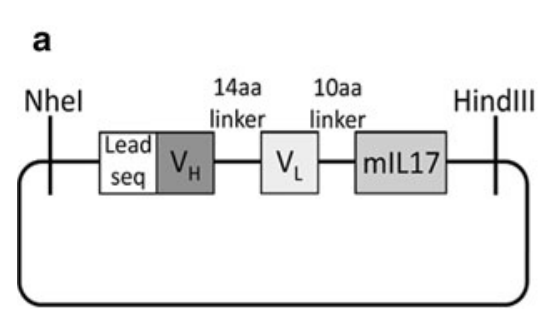

d

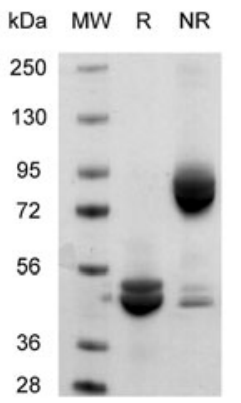

b

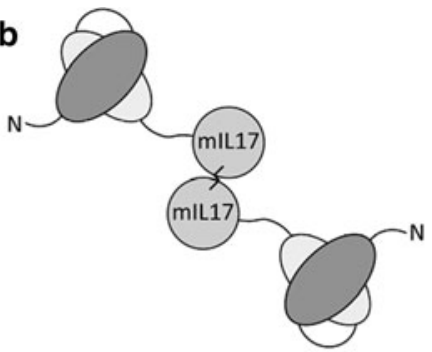

e

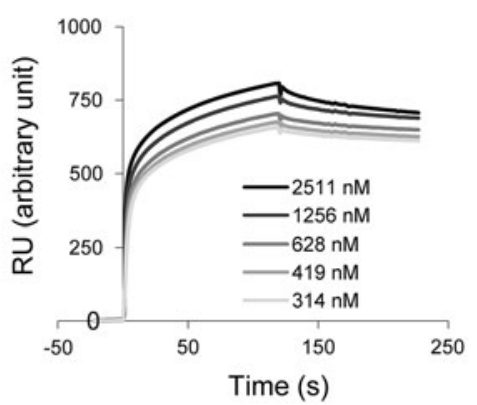

h

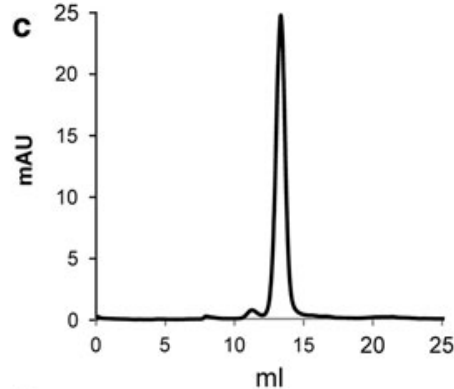

f

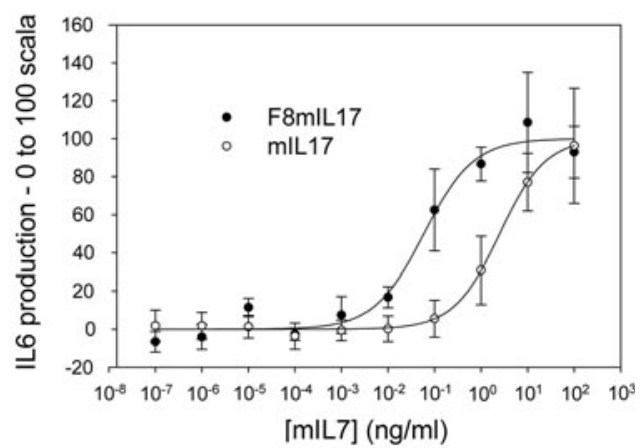

g

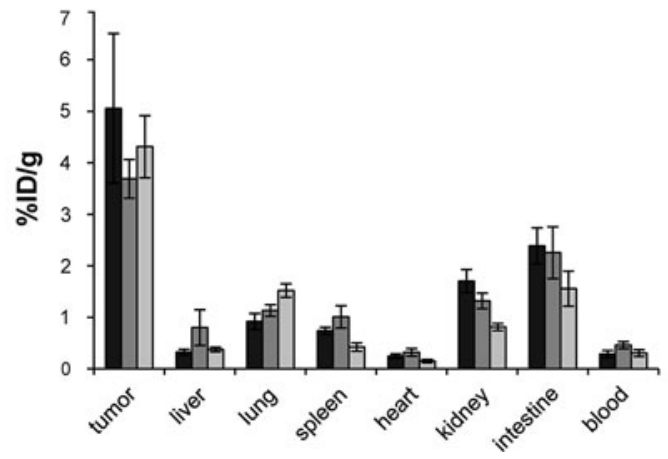

Fig. 1 Cloning, expression and characterization of F8-mIL17. a Schematic representation of the cloning strategy of F8-mIL17 with a 14-amino acid linker between variable heavy (VH) and light (VL) chain. b Schematic representation of the domain assembly of F8mIL17. c Gel filtration analysis of affinity-purified F8-mIL17. The peak corresponds to the homodimeric form of the fusion protein. d SDS-PAGE analysis of purified F8-mIL17: molecular weights (MW), reducing (R) and non reducing (NR) conditions. The fusion protein is present in different glycosylation forms. e BIAcore analysis of F8-mIL17 on EDA-coated chip. f IL6 production bioactivity assay on NIH 3T3 fibroblasts. F8-mIL17 displayed biological activity comparable with the one of recombinant murine IL17 (mean of 3 replicates $\pm \mathrm{SD}$ ). $\mathrm{g}$ Tumor targeting properties of F8-mIL17 in
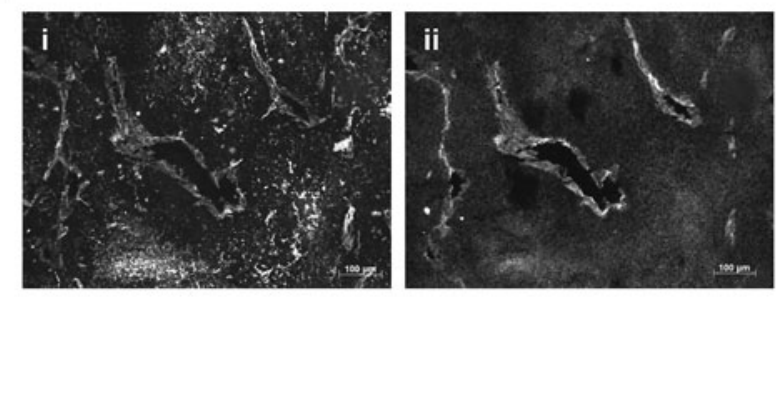

biodistribution studies in Balb/c nude and 129/SvPas mice $(n=5)$ bearing subcutaneous F9 tumors. $14.5 \mu \mathrm{g}$ radiolabeled ${ }^{125} \mathrm{I}-\mathrm{F} 8-\mathrm{mIL} 17$ were injected i.v in Balb/c nude (black square) and 129/SvPas mice (grey square), $7.5 \mu \mathrm{g}$ radiolabeled ${ }^{125} \mathrm{I}-\mathrm{F} 8-\mathrm{mIL} 17$ and $60 \mu \mathrm{g}$ unlabeled F8-mIL17 were mixed and injected i.v. in 129/SvPas mice (light grey square), which were sacrificed after $24 \mathrm{~h}$. Organs were excised and radioactivity counted, expressing results as percent injected dose per gram of tissue $(\% \mathrm{ID} / \mathrm{g}) \pm \mathrm{SE}$. A selective accumulation of F8-mIL17 in F9 tumors could be observed over time. h Immunofluorescent staining of F8-mIL17 on F9 tumors. F8-mIL17 was biotinylated and used as staining reagent in an immunofluorescence procedure. F8-mIL17 shows a vascular staining pattern. Scale bars $100 \mu \mathrm{m}$ 
When administered intravenously at doses of $100 \mu \mathrm{g}$ (day 5, 7, 9), F8-mIL17 displayed no anti-cancer activity in immunocompetent (Fig. 2a) and athymic mice (Fig. 2b) bearing subcutaneous F9 tumors, compared to the saline control group. There was no weight loss in immunocompetent mice, but up to $15 \%$ weight loss in nude mice [Supplementary Figure 1]. Figure 2c shows a representative immunofluorescence analysis of the tumor masses at the end of the therapy. Staining of the tumors with an anti-CD45 antibody (Fig. 2ci, vi), F4/80 antibody (Fig. 2cii, vii), Asialo-GM1 antibody (Fig. 2ciii, viii) and CD4 antibody (Fig. 2civ, ix) revealed a higher leukocytes, macrophages, NK cells and CD4 T cells (Fig. 2civ, ix) infiltration in the tumors treated with F8-mIL17 (Fig. 2ciiv), compared to the saline control group (Fig. 2cvi-ix).
By contrast, CD8 T cells were found to be low in both treatment groups (Fig. 2cv, $\mathrm{x}$ ) (more pictures are available in Supplementary Figures 2-6). Figure 2d shows representative images (the experiment was repeated twice) of tumor blood vessels 3 days after the last injection, from mice treated with F8-mIL17 (Fig. 2di-vi) or with saline (Fig. 2dvii-xii). F8-mIL17 treatment resulted in a significant $(P<0.01)$ increase in blood vessel density (Fig. 2e).

We can conclude that the antibody-based targeted delivery of mIL17 to the tumor blood vessels enhances tumor angiogenesis and leukocyte infiltration into the tumor mass, but this (in contrast to fusion proteins based on other cytokines such as IL2, IL7, IL12, IL15, GM-CSF, TNF [12, 13, 15-20, 24]) does not lead to a measurable anti-cancer activity in immunocompetent or in nude mice. a

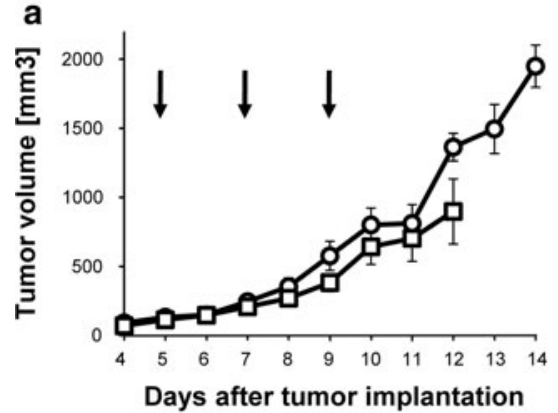

d

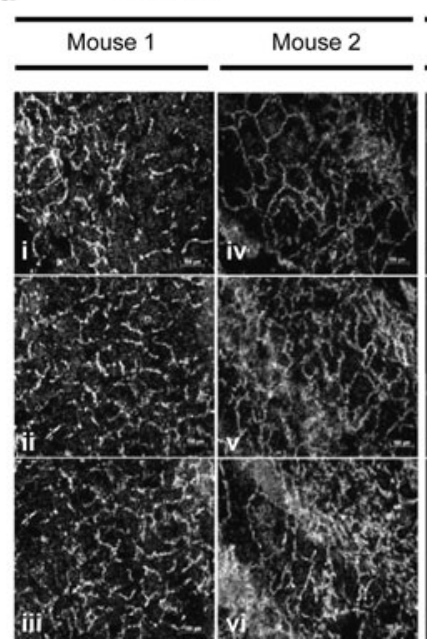

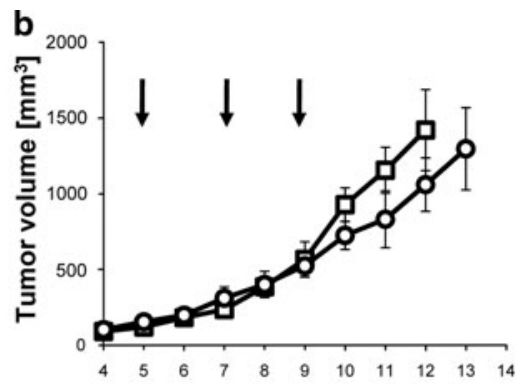

Days after tumor implantation

saline
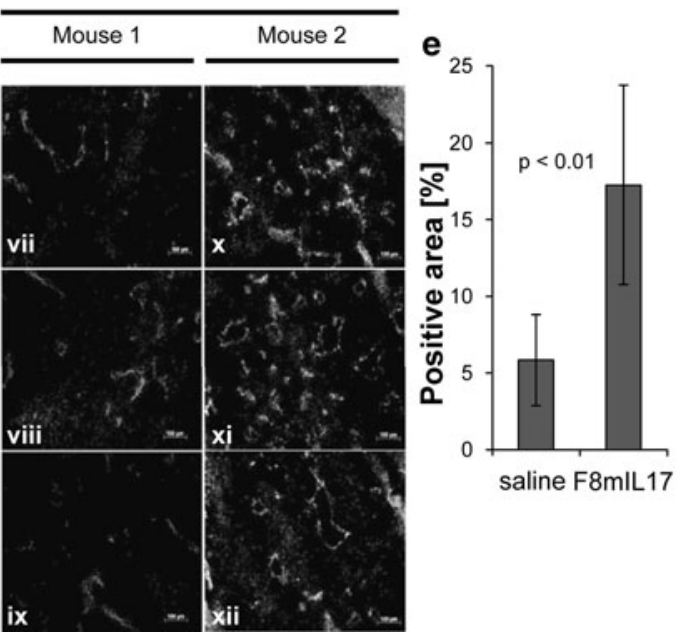

C
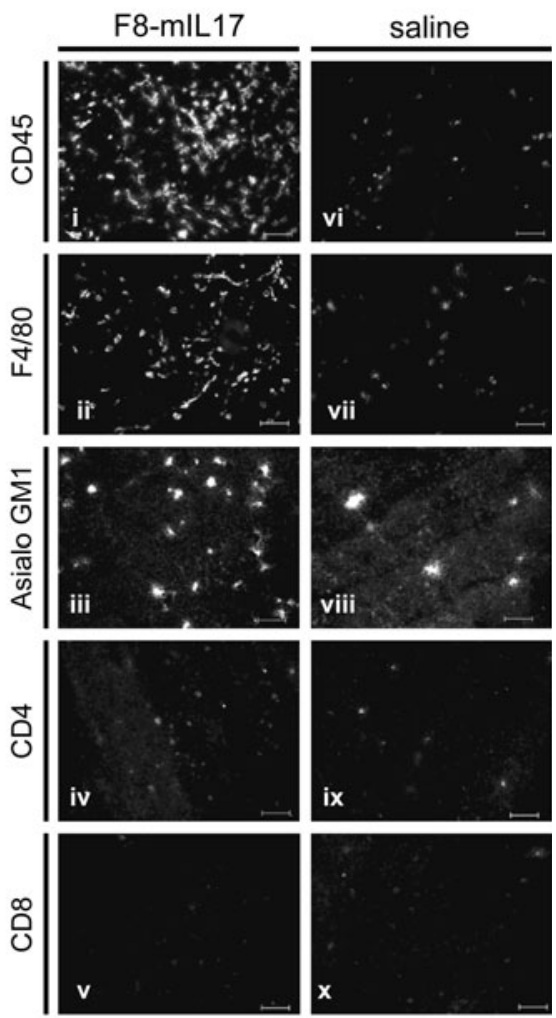

Fig. 2 Effects of F8-mIL17 in mice bearing subcutaneously-grafted F9 tumors. Tumor growth curves of a immunocompetent 129/SvPas or $\mathbf{b}$ athymic Balb/c nude mice bearing F9 tumors after i.v. treatment with three injections of $100 \mu \mathrm{g} \mathrm{F8-mIL17} \mathrm{(square;} n=5, n=5$ ) and saline (circle; $n=3, n=4$ ). Days of treatment (day 5, 7, 9 after tumor implantation) are indicated by arrows. Data represent mean tumor volumes $\pm \mathrm{SE}$. c Immunofluorescence analysis of tumor-infiltrating immune cells. Tumor sections of therapy mice were stained for leukocytes (CD45), macrophages (F4/80), NK cells (Asialo/GM1), CD4 T cells and CD8 T cells. Scale bars $=100 \mu \mathrm{m}$. Representative images of leukocytes $(i, v i)$, macrophages $(i i, v i i)$, NK cells (iii, viii), CD4 T cells $(i v, i x)$ and CD8 T cells $(v, x)$ staining in F8mIL17 $(i-v)$ and saline $(v i-x)$ treated tumors. d Immunofluorescence analysis of vasculature (CD31 staining). Immunocompetent F9 tumor-bearing mice were injected i.v. three times every $48 \mathrm{~h}$ with $100 \mu \mathrm{g}$ F8-mIL17 or saline. Mice were sacrificed 3 days after last injection. Tumor sections were stained for endothelial cells. Representative images of 2 mice treated with F8-mIL17 ( $i-i i i, i v-v i)$ and 2 mice treated with saline (vii-ix, $x$-xii). e F9 tumor sections were evaluated for area percentage positive staining using ImageJ $(P<0.01$, Student $t$ test $)$ 
Our findings are consistent with previous publications, in which no difference in tumor growth rate was observed in IL17 -/- mice compared to wild-type mice [11], or in which tumor cells transfected with mIL17 displayed no anti-cancer activity $[6,7,9]$. It remains to be seen whether the activity of IL17 could be different in an orthotopic setting. In spite of these considerations, it would be conceivable to use IL17-based immunocytokines to stimulate therapeutic angiogenesis at sites of disease [26].

Acknowledgments We thank MD Michael Fiechter for help with therapy experiments and Dr. Giancarlo Marra for kindly providing NIH 3T3 fibroblasts. Financial contributions from the Swiss National Science Foundation, the ETH Zürich, the European Union (ADAMANT Project), the Swiss Cancer League, the Swiss-Bridge Foundation and the Stammbach Foundation are gratefully acknowledged.

Conflict of interest DN is founder and shareholder of Philogen, a biotech company that owns the F8 antibody.

\section{References}

1. Murugaiyan G, Saha B (2009) Protumor vs antitumor functions of IL-17. J Immunol 183(7):4169-4175

2. Gaffen SL (2004) Biology of recently discovered cytokines: interleukin-17-a unique inflammatory cytokine with roles in bone biology and arthritis. Arthritis Res Ther 6(6):240-247

3. Onishi RM, Gaffen SL (2010) Interleukin-17 and its target genes: mechanisms of interleukin-17 function in disease. Immunology 129 (3):311-321

4. Yu JJ, Gaffen SL (2008) Interleukin-17: a novel inflammatory cytokine that bridges innate and adaptive immunity. Front Biosci 13:170-177

5. Gaffen SL (2008) An overview of IL-17 function and signaling. Cytokine 43(3):402-407

6. Numasaki M, Watanabe M, Suzuki T, Takahashi H, Nakamura A, McAllister F, Hishinuma T, Goto J, Lotze MT, Kolls JK, Sasaki $\mathrm{H}$ (2005) IL-17 enhances the net angiogenic activity and in vivo growth of human non-small cell lung cancer in SCID mice through promoting CXCR-2-dependent angiogenesis. J Immunol 175(9):6177-6189

7. Tartour E, Fossiez F, Joyeux I, Galinha A, Gey A, Claret E, Sastre-Garau X, Couturier J, Mosseri V, Vives V, Banchereau J, Fridman WH, Wijdenes J, Lebecque S, Sautes-Fridman C (1999) Interleukin 17, a T-cell-derived cytokine, promotes tumorigenicity of human cervical tumors in nude mice. Cancer Res 59(15):3698-3704

8. Benchetrit F, Ciree A, Vives V, Warnier G, Gey A, SautesFridman C, Fossiez F, Haicheur N, Fridman WH, Tartour E (2002) Interleukin-17 inhibits tumor cell growth by means of a T-cell-dependent mechanism. Blood 99(6):2114-2121

9. Numasaki M, Fukushi J, Ono M, Narula SK, Zavodny PJ, Kudo T, Robbins PD, Tahara H, Lotze MT (2003) Interleukin-17 promotes angiogenesis and tumor growth. Blood 101(7):2620-2627

10. Kryczek I, Wei S, Szeliga W, Vatan L, Zou W (2009) Endogenous IL-17 contributes to reduced tumor growth and metastasis. Blood 114(2):357-359
11. Ngiow SF, Smyth MJ, Teng MW (2010) Does IL-17 suppress tumor growth? Blood 115 (12):2554-2555; author reply 2556-2557

12. Kaspar M, Trachsel E, Neri D (2007) The antibody-mediated targeted delivery of interleukin-15 and GM-CSF to the tumor neovasculature inhibits tumor growth and metastasis. Cancer Res 67(10):4940-4948

13. Schliemann C, Palumbo A, Zuberbuhler K, Villa A, Kaspar M, Trachsel E, Klapper W, Menssen HD, Neri D (2009) Complete eradication of human B-cell lymphoma xenografts using rituximab in combination with the immunocytokine L19-IL2. Blood 113(10):2275-2283

14. Ebbinghaus C, Ronca R, Kaspar M, Grabulovski D, Berndt A, Kosmehl H, Zardi L, Neri D (2005) Engineered vascular-targeting antibody-interferon-gamma fusion protein for cancer therapy. Int J Cancer 116(2):304-313

15. Halin C, Rondini S, Nilsson F, Berndt A, Kosmehl H, Zardi L, Neri D (2002) Enhancement of the antitumor activity of interleukin-12 by targeted delivery to neovasculature. Nat Biotechnol 20(3):264-269

16. Carnemolla B, Borsi L, Balza E, Castellani P, Meazza R, Berndt A, Ferrini S, Kosmehl H, Neri D, Zardi L (2002) Enhancement of the antitumor properties of interleukin-2 by its targeted delivery to the tumor blood vessel extracellular matrix. Blood 99(5):1659-1665

17. Borsi L, Balza E, Carnemolla B, Sassi F, Castellani P, Berndt A, Kosmehl H, Biro A, Siri A, Orecchia P, Grassi J, Neri D, Zardi L (2003) Selective targeted delivery of TNFalpha to tumor blood vessels. Blood 102(13):4384-4392

18. Halin C, Gafner V, Villani ME, Borsi L, Berndt A, Kosmehl H, Zardi L, Neri D (2003) Synergistic therapeutic effects of a tumor targeting antibody fragment, fused to interleukin 12 and to tumor necrosis factor alpha. Cancer Res 63(12):3202-3210

19. Marlind J, Kaspar M, Trachsel E, Sommavilla R, Hindle S, Bacci C, Giovannoni L, Neri D (2008) Antibody-mediated delivery of interleukin-2 to the stroma of breast cancer strongly enhances the potency of chemotherapy. Clin Cancer Res 14(20):6515-6524

20. Pedretti M, Verpelli C, Marlind J, Bertani G, Sala C, Neri D, Bello L (2010) Combination of temozolomide with immunocytokine F16-IL2 for the treatment of glioblastoma. Br J Cancer 103(6):827-836

21. Rybak JN, Roesli C, Kaspar M, Villa A, Neri D (2007) The extradomain A of fibronectin is a vascular marker of solid tumors and metastases. Cancer Res 67(22):10948-10957

22. Frey K, Zivanovic A, Schwager K, Neri D (2011) Antibodybased targeting of interferon-alpha to the tumor neovasculature: a critical evaluation. Integr Biol (Camb) 3(4):468-478

23. Villa A, Trachsel E, Kaspar M, Schliemann C, Sommavilla R, Rybak JN, Rosli C, Borsi L, Neri D (2008) A high-affinity human monoclonal antibody specific to the alternatively spliced EDA domain of fibronectin efficiently targets tumor neo-vasculature in vivo. Int J Cancer 122(11):2405-2413

24. Pasche N, Woytschak J, Wulhfard S, Villa A, Frey K, Neri D (2011) Cloning and characterization of novel tumor-targeting immunocytokines based on murine IL7. J Biotechnol 154(1): 84-92

25. Sommavilla R, Pasche N, Trachsel E, Giovannoni L, Roesli C, Villa A, Neri D, Kaspar M (2010) Expression, engineering and characterization of the tumor-targeting heterodimeric immunocytokine F8-IL12. Protein Eng Des Sel 23(8):653-661

26. Dor Y, Djonov V, Keshet E (2003) Induction of vascular networks in adult organs: implications to proangiogenic therapy. Ann N Y Acad Sci 995:208-216 